\section{Influence of Temperature upon Blood Coagulation in a Cold- and a Warm- blooded Animal}

THe influence of change of temperature upon the speed of blood clotting in warm-blooded animals is well recognized. Indeed, the fact that human plasma at $0^{\circ} \mathrm{C}$. does not clot for several hours is utilized in some investigations in coagulation research.

If a similar depression of blood coagulation at low temperatures occurred in cold-blooded animals this would tend to make them unusually vulnerable to trauma during some part of their lives. This consideration led us to compare the influence of tem. perature on the clotting mechanism of a warmand a cold-blooded animal.

Because of its ready availability, human blood was compared with that of the South African clawed toad Xenopus laevis. Human blood was collected by venepuncture and toad blood was obtained from the heart after the animal had been anæsthetized with ether. Considerable difficulty was at first experienced in obtaining adequate unclotted samples by methods involving direct cardiac puncture. To overcome this, the heart was exposed by a central skin excision, division of the pectoral girdle and opening of the pericardium. The animal was then placed on a wire netting (approximately $1 \mathrm{~cm}^{2}$ network) with the heart dependent between the wires. In this way approximately $3 \mathrm{ml}$. could be collected into an underlying vessel within a few seconds of a doep scalpel incision into the ventricle. The contamination by tissue juices, although present, is probably minimal under these conditions and the sample appears preferable to that obtained after a lengthy and technically difficult cardiac puncture technique. The blood from three toads was pooled to make a single specimen.

All samples were collected in graduated siliconed centrifuge tubes with 3.8 per cent of sodium citrate in a proportion of nine parts of blood to one part of eitrate solution.

The thromboplastin preparations were made from emulsified human brain and emulsified toad brain and spinal cord. The dilutions were adjusted to give a satisfactory one-stage prothrombin time with human and toad plasma respectively. The thromboplastin

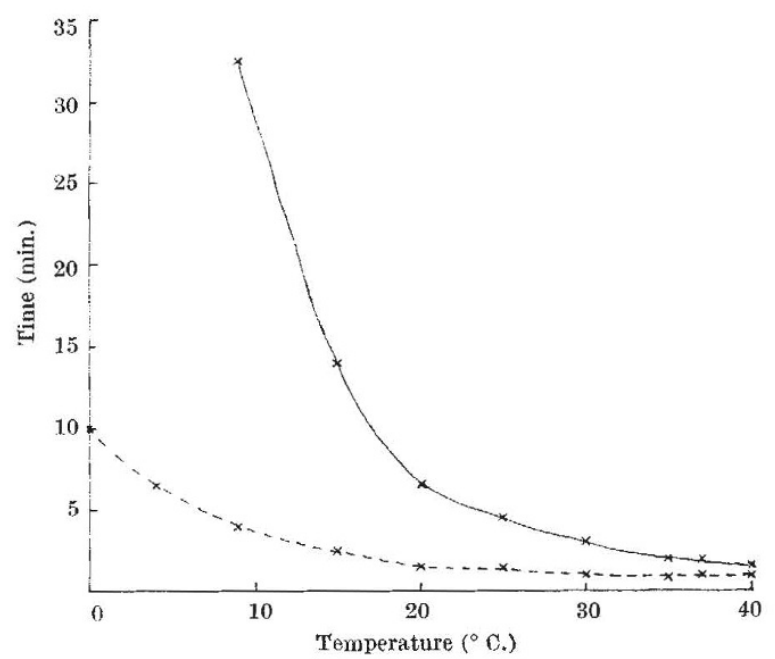

Fig. 1. The calcium clotting time (whole blood). - - human;

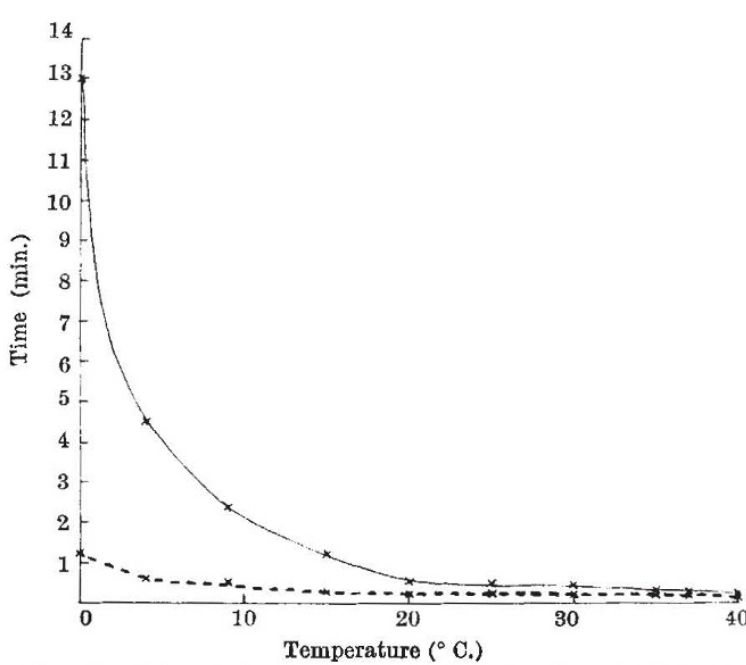

Fig. 2. Thromboplastin time (one-stage 'prothrombin' time). - , human; - - - toad

time (one-stage 'prothrombin' time) and the calcium clotting time estimations were performed as recommended by Biggs and Macfarlan ${ }^{1}$, but in the latter test citrated whole blood was substituted for citrated plasma.

The results are shown in Figs. 1 and 2.

A determination of calcium clotting time was used in preference to one of whole-blood clotting time, because the latter would have required several fresh samples of blood, needing more than a single animal to cover the complete range of temperature being investigated. It therefore appeared desirable to test the clotting time of a single pooled citrated sample obtained from three animals after recalcifying at various temperatures.

In order to keep the platelet activity of the specimens as close to physiological conditions as possible, citrated whole blood rather than plasma was used in the estimations of the clotting time.

Quick $^{2}$ showed that tissue thromboplastin of a reptile (turtle) showed species specificity, when mixed with the plasma of warm-blooded animals. This specificity has been demonstrated with the amphibian used in this investigation. The thromboplastin times of human or toad plasma were therefore compared using human and toad brain emulsions respectively. Owing to the small size of the toad brain, the spinal cord was also included in the preparations.

From a comparison of the two curves in Fig. $l$ it can be seen that the toad-clotting mechanism appears well adapted for functioning at low temperatures. Fig. 2 shows the influence of temperature on the thromboplastin time, which does not involve certain clotting factors, such as hæmophilic globulin, which are needed for active intrinsic thromboplastin formation. As with the recalcified whole blood clotting time the thromboplastin time in the cold-blooded animal appears comparatively unaffected by a temperature lowered below $10^{\circ} \mathrm{C}$.

Massachusetts General Hospital,

$$
\text { H. B. Anstatll }
$$

Boston, Massachusetts.

$$
\text { R. G. Huntsman }
$$

Department of Clinical Pathology,

Guy's Hospital, London, S.E.1.

'Biggs, R., and Macfarlane, R. G., "Human Blood Coagulation and its Disorders" (Blackwell Scientiflc Publications, Oxford, 1953), 2Quick, A. J., J. Biol. Chem., 123, xcix (1938). 